\title{
IMPROVEMENT OF SOME ECONOMIC CHRACTERS THROUGH VARIATION INDUCED VIA MUTAGENESIS IN SUMMER SQUASH \\ El-Miniawy, S.M. ${ }^{1}$; M.E. Ragab'; Kh.A. Soliman' ${ }^{2}$ and A.M. Asbah' \\ 1-Department of Horticulture, Faculty of Agriculture, Ain Shams \\ University, Shoubra-Elkeima, Cairo, Egypt \\ 2-Department of Genetics, Faculty of Agriculture, Ain Shams University, Shoubra-Elkeima, Cairo Egypt
}

\begin{abstract}
This work was carried out at the Experimental Farm of Faculty of Agriculture, Ain Shams University, at Shalakan, in the fall and summer seasons of 2004/2005 and 2005/2006. The aim of this experiment was to study the mutagenesis effect of both gamma irradiation (at doses 10,20,30, and $40 \mathrm{kr}$ ) and EMS (at concentrations 0.025, $0.05,0.1$, and $0.2 \%$ ) on two summer squash cultivars, Eskandarani and Gabla, as an attempt to induce mutations of valuable productivity. Three mutant generations were carried out after treating with the two mutagenesis and the results indicated that in $\mathrm{M} 1$ generation the values of most studied traits was decreased at the high doses and high concentrations of gamma-rays and EMS, respectively, compared with control plants. In M2-generation, five types of mutant were selected, these mutants were different from their control plant. In M3-generation, four type mutants, namely early flowering, node position of first pistillate flower, high number of pistillate flower and large fruit selected from the M2-generation, were raised to study their behavior compared to the control plants. The progeny test of these mutants revealed that all the mutants in M3 retained the behavior they show in the M2-generation. These mutants were superior to their control plant in these economic characters.
\end{abstract}

Keywords: summer squash, gamma rays, EMS, mutants, mutagenesis

\section{INTRODUCTION}

Summer squash (Cucurbita pepo. L.) is one of the most popular vegetable crops in most Arab countries. However, there are only two local cultivars of squash in Egypt. Balady, which is lately discarded for its prostrate growth habit and low yield and Eskandarani, which is highly yielding and satisfies both the producer and consumer. There are littlie studies on using mutagenesis to induce mutation in summer squash or Cucurbitaceae family, therefore literature concerning other crops will be reviewed. Shalaby et al. (1983) soaked cloves of Egyptian garlic with EMS solution at concentrations of $0.0,0.025,0.05,0.1$ or $0.2 \%$ for $12,24,48$ and 72 hours. The result showed that, in the M1-generation, both higher concentrations and longer periods of soaking had inhibitory effect on bulb diameter, bulb weight, number of cloves per bulb, average clove weight and total yield. Saxena and Solanki (1986) irradiated seeds of tomato cv. Pusa Ruby to different doses of gamma-rays,i.e., 5, 10, 15, 20 and $25 \mathrm{Kr}$. They stated that doses lower than $20 \mathrm{Kr}$ induced early flowering and fruiting with an appreciable effect in $5 \mathrm{Kr}$ doses. Tickoo (1987) studied the effect of EMS for inducing mutations in mung bean (Vigna radiate L.). In M2-generation, macromutations identified 
were dwarf, many pods, large pods, long pods, white pods, large seeds and seed coat color. The mutants bred true in the M3-generation. Nofal (1989) studied the effect of gamma-ray doses $(5,10$ and $20 \mathrm{Kr})$ on two pea cultivars. It was found that all doses significantly reduced the mean number of pods per plant of both cultivars in M1-generation. Hassan (1991) treated seeds of lupine with EMS at concentration of $0.1,0.15,0.2$ and $0.25 \%$. It was reported that the variability of such trait as indicated by C.V.\% values showed elevation above that of the control. Greater variability in number of pods per plant compared with control was showed by Ahmad and Yagoob(1993). Kumar and Mishra (1999) found in Vigna radiate L. that dry seeds treated with varying doses of gamma-rays $(10,20,30$ and $40 \mathrm{Kr})$ gave reduced number of leaves per plant, but increased the number of days to flowering, on mung bean with 10 to $30 \mathrm{Kr}$ gamma-rays. Srivastava and Singh (1996) studied the effect of different EMS concentrations (0.1, 0.2 , and $0.3 \%$ ) on two pigeon pea cultivars. They isolated two kind of mutants; high yield and high protein content in M2-generation. These mutants were further taken to M3 and M4 generations where they were found to be bred true.

The aim of this experiment was to study the mutagenesis effect of both gamma irradiation and EMS on two summer squash cultivars, Eskandarani and Gabla, as an attempt to induce mutations of valuable productivity.

\section{MATERIALS AND METHODS}

This work was carried out at the Experimental Farm of Faculty of Agriculture, Ain Shams University, at Shalakan, in the fall and summer seasons of 2004-2005 and 2005-2006. Seeds of two cultivars (pure line) of summer squash (Cucurbita pepo L.), namely Eskandarani and Gabla, were exposed to different doses of gamma rays, i.e., 0, 10, 20, 30 and $40 \mathrm{Kr}$, from a cobalt-60 source at the Middle Easterm Regional Radioisotope Center of the Arab Countries, Dokki, Cairo, Egypt. Dose rate was 3.80 and 4.21 $\mathrm{rad} / \mathrm{sec}$, in the first and second season, respectively. Dry seeds of the two summer squash cultivars were treated with freshly prepared aqueous solution of ethylmethane sulphonate (EMS) at the concentrations $0.0,0.025,0.05,0.1$ and $0.2 \%$ for 12 hours at room temperature. The soaked seeds were thoroughly washed in running water to remove the mutagen. Immediately after the treatment, the seeds were sown in field. Seeds soaked in a tap water under the above mentioned conditions were used as control. The treatments with two mutagenesis were done on August 2 and 3 in 2004 and 2005 seasons, respectively.

M1-generation:

Treated and non-treated seeds (control) from the two cultivars were sown in four replications and the complete randomized block design was used in M1-generation.

Each experimental plot consisted of four rows with 5 meter length and 0.7 meter width. Two seeds were planted in each hill, a distance of $50 \mathrm{~cm}$ was maintained between the plants, and the field was then irrigated. The plants were thinned to a single seedling per hill two weeks after germination. 
All replications received similar treatments as regards to cultivation, manuring, irrigation, pest and disease control, and other agricultural practices as commonly followed in the district. The following data were recorded:

1- Number of days from sowing till the first pistillate flower opening.

2- Number of staminate flower per plant.

3- Number of pistillate flowers per plant.

4- Sex ratio

5- Early and total yield: Fruits were harvested at two-day intervals. Early and total yields were calculated, as fruit weight per plot and number of fruits per plant. Fruits harvested during 15 days from the beginning of harvest were considered as early yield.

\section{M2-generation:}

Random seeds from the individual M1-plant of each treatment were planted as a family on February 15, 2005. Seeds of Every family were planted in 15 rows with 5 meter long and $70 \mathrm{~cm}$ wide and $50 \mathrm{~cm}$ apart, beside two rows of untreated seeds as a control for each treatment.

During the growth period, macromutations such as dwarf, vigorous plants, number of days to first pisillate flower, number of nodes to first pistillate flower, fruit length, fruit diameter and other morphplogical abnormalities or any plant showing chimeras were recorded.

Data recorded:

1. Number of days from sowing till the first pistillate flower opening.

2. Number of nodes till first pistillate flower.

3. Number of pistillate flowers per plant.

4. Average fruit weight without fruit neck.

5. Number of fruits per plant.

Seeds of each fertile mutant plant were kept separately in paper bag for sowing in next season (2006) to raise M3-generation.

M3-generation:

Seeds of the best mutant plants, which were selected from M2generation, were sown on $15^{\text {th }}$ of February, 2006, to provide the experimental plant materials of M3-generation. The progeny of each M2-deviant plant was grown as a single family in three rows each one considered as replicate, the length of row was 7 meter and the width was $70 \mathrm{~cm}$ and $50 \mathrm{~cm}$ apart between plants. Control seeds were, also, sown in the same designs.

From each replicate 4 plants were determined and the following data were recorded:

1. Stem length: the plants were picked at the end of season and the average of stem length was measured.

2. Average number of leaves per plant: The plants were dug out at the end of season and the leaf number was counted.

3. Number of days from sowing till the first pistillate flower opening.

4. Number of nodes till first pistillate flower.

5. Number of staminate flowers per plant.

6. Number of pistillate flowers per plant.

7. Average fruit length was measured without fruit neck

8. Average fruit diameter was measured at the middle of fruit.

9. Average fruit weight was measured without neck. 
10. Number of fruits per plant.

11 . Weight of 100 seeds.

\section{Statistical analysis:}

\section{M1 and M3generations}

Data were statistically analyzed according to the regular analysis of variance of complete randomized block design. Duncan,s multiple range test was used for the comparison between treatment means (Waller and Duncan, 1960)

\section{M2-generation}

Data on M2-micro-mutation were statistically analyzed by calculating minimum value, maximum value(range of variability), the means of treatment $(X)$, variance (S2), standard error (S.E), coefficient variation (CV \%) for each treatment.

\section{RESULTS AND DESCOSION}

\section{M1-generation}

Number of days for first pistillate flower:

Data presented in Table (1) show that in both cultivars the low dose of gamma rays $(10 \mathrm{Kr})$ decreased the period from sowing to flowering. While, the high doses of two mutagenesis (30 and $40 \mathrm{Kr}$ ) or (0.1 and $0.2 \% \mathrm{EMS}$ ) increased such period. In both cultivars the lowest values for number of days for first pistillate flower was at $10 \mathrm{Kr}$ of gamma-rays. Early flowering could be attributed to that low doses of irradiation, generally stimulate cell growth, increase the rate of growth and produce early flowering in specific case and at some growing stage according to radiation type and dose as well as to plant species ( Abd El-Maksoud, 1980). Also, it can be explained as a result of delaying or inhibiting the synthesis of florigens which resulted in delaying flowering under the external environmental conditions because induction of flowering can be affected by many factors, either internal or external. These results are in agreement with those of Saxena and Solanki (1986) and Kumar and Mishra (1999).

Number of pistillate and staminate flowers and sex ratio:

From Table (1) we can notice that in Eskandarani cultivar high doses of gamma-rays decreased the number of staminate and pistillate flowers and increased the sex ratio, while the low dose $1 \mathrm{Kr}$ increased the number of pistillate flowers and decreased the sex ratio. In the same cultivar, EMS caused more decreasing in number of pistillate flowers and more increasing in sex ratio compared with gamma-rays. On the other hand, in Gabla cultivar all concentrations of EMS and high doses of gamma- rays increased the number of staminate flowers and sex ratio, while $10 \mathrm{Kr}$ of gamma-rays increased the number of pistillate flowers and decreased the sex ratio. The changing in number of flowers and sex ratio due to some treatments may be returned to the stimulation effect of these treatments on plant growth and growth regulator in the plant.

Yield and yield components:

Results presented in Table (2) showed that the low and medium doses of EMS did not affect the early and total yield of the two cultivars in the both seasons, while the high doses of EMS $(0.1,0.2)$ caused high significant 
decrease in the early and total yield of two cultivars in both seasons. The10 $\mathrm{Kr}$ dose of gamma-rays caused increasing in early and total yield in both cultivars, while the higher doses of gamma-rays caused significant decrease in early and total yield. The increasing in early and total yield at $10 \mathrm{Kr}$ dose may be returned to the increasing in vegetative growth, earlier in flowering, number of pistillate flowers and fruit weight at this dose as we showed previously. While the decreasing in early and total yield at high doses of two mutagenesis maybe returned to the decreasing in plant growth, number of pistillate flower and fruit weight at these doses. These results are confirmed with those of Shalaby et al. (1983) and Nofal 1989.

Table (1): Effect of gamma rays and EMS on some flowering traits of two summer squash cultivars in M1- generation in 2004 and2005 seasons.

\begin{tabular}{|c|c|c|c|c|c|c|c|c|}
\hline Treatments & $\begin{array}{l}\text { No. of } \\
\text { days for } \\
\text { first } \\
\text { pistillate } \\
\text { flower }\end{array}$ & $\begin{array}{c}\text { No. of } \\
\text { staminate } \\
\text { flowers/ } \\
\text { plant }\end{array}$ & $\begin{array}{c}\text { No. of } \\
\text { pistillate } \\
\text { flowers/ } \\
\text { plant }\end{array}$ & $\begin{array}{c}\text { Sex } \\
\text { ratio }\end{array}$ & $\begin{array}{l}\text { No. of } \\
\text { days for } \\
\text { first } \\
\text { pistillate } \\
\text { flower }\end{array}$ & $\begin{array}{c}\text { No. of } \\
\text { staminate } \\
\text { flowers } / \\
\text { plant }\end{array}$ & $\begin{array}{c}\text { No. of } \\
\text { pistillate } \\
\text { flowers/ } \\
\text { plant }\end{array}$ & Sex ratio \\
\hline \multicolumn{5}{|c|}{2004} & \multicolumn{4}{|c|}{2005} \\
\hline \multicolumn{9}{|l|}{ Eskandarani } \\
\hline Control & $45.10 \mathrm{H}$ & 10.11 DEF & $11.40 \mathrm{E}$ & $0.88 \mathrm{EFG}$ & $42.58 \mathrm{FGH}$ & $9.36 \mathrm{BCD}$ & $10.97 \mathrm{DE}$ & $0.86 \mathrm{FGH}$ \\
\hline $0.025 \%$ & $46.38 \mathrm{FG}$ & $11.06 \mathrm{BCD}$ & $11.94 \mathrm{DE}$ & $1.03 \mathrm{CD}$ & $43.75 \mathrm{EFG}$ & $9.55 \mathrm{BCD}$ & 9.77FGGH & 0.97 DEF \\
\hline $0.05 \%$ & $47.97 \mathrm{E}$ & $11.53 \mathrm{ABC}$ & $9.95 \mathrm{FG}$ & $1.14 \mathrm{~B}$ & $44.55 \mathrm{EF}$ & 9.72 BCD & $8.50 \mathrm{HI}$ & $1.15 \mathrm{CD}$ \\
\hline $0.1 \%$ & $51.93 \mathrm{D}$ & $10.56 \mathrm{CDE}$ & 8.701 & $1.21 \mathrm{~B}$ & $47.99 \mathrm{CD}$ & $9.08 \mathrm{CDE}$ & $7.09 \mathrm{~J}$ & $1.27 \mathrm{BC}$ \\
\hline $2 \%$ & $54.91 \mathrm{C}$ & $10.52 \mathrm{CDE}$ & & $1.36 \mathrm{~A}$ & $51.47 \mathrm{~B}$ & 8.34 CDEF & $5 \mathrm{~K}$ & $1.68 \mathrm{~A}$ \\
\hline $10 \mathrm{Kr}$ & $40.66 \mathrm{~K}$ & $9.75 \mathrm{DEFG}$ & $13.36 \mathrm{~B}$ & $0.72 \mathrm{HI}$ & $39.58 \mathrm{IJ}$ & $9.23 \mathrm{BCDE}$ & $12.70 \mathrm{BC}$ & $0.72 \mathrm{GHI}$ \\
\hline $20 \mathrm{Kr}$ & $47.54 \mathrm{EF}$ & $9.48 \mathrm{DEF}$ & $11.54 \mathrm{E}$ & $0.85 \mathrm{FG}$ & $45.89 \mathrm{DE}$ & $8.91 \mathrm{CDEF}$ & $9.91 \mathrm{EF}$ & OEFG \\
\hline $30 \mathrm{Kr}$ & $56.50 \mathrm{~B}$ & $8.37 \mathrm{GH}$ & $9.19 \mathrm{HI}$ & $0.92 \mathrm{DEF}$ & $53.97 \mathrm{~A}$ & 7.74 DEF & $8.54 \mathrm{GHI}$ & $0.98 \mathrm{DEF}$ \\
\hline $40 \mathrm{Kr}$ & $58.81 \mathrm{~A}$ & $7.69 \mathrm{H}$ & $7.02 \mathrm{~K}$ & 1.12 BC & $55.42 \mathrm{~A}$ & $6.95 \mathrm{~F}$ & $6.78 \mathrm{~J}$ & $1.12 \mathrm{CD}$ \\
\hline \multicolumn{9}{|l|}{ Gabla } \\
\hline Control & $40.64 \mathrm{~K}$ & $8.90 \mathrm{FGH}$ & $13.65 \mathrm{~B}$ & $0.64 I$ & $37.89 \mathrm{~J}$ & $9.05 \mathrm{CDE}$ & $14.54 \mathrm{~A}$ & $0.61 \mathrm{IJ}$ \\
\hline $0.025 \%$ & $41.74 \mathrm{JK}$ & $12.84 \mathrm{~A}$ & $13.35 \mathrm{~B}$ & $0.95 \mathrm{DEF}$ & $41.45 \mathrm{GHI}$ & $12.31 \mathrm{~A}$ & $12.77 \mathrm{BC}$ & $0.97 \mathrm{DEF}$ \\
\hline $0.05 \%$ & $44.75 \mathrm{H}$ & $12.38 \mathrm{AB}$ & $12.73 \mathrm{C}$ & $0.96 \mathrm{DE}$ & $43.46 \mathrm{EFG}$ & $13.14 \mathrm{~A}$ & $11.78 \mathrm{CD}$ & $1.10 \mathrm{CDE}$ \\
\hline & $46.72 \mathrm{FG}$ & $11.69 \mathrm{ABC}$ & $10.41 \mathrm{~F}$ & $1.10 \mathrm{BC}$ & $45.56 \mathrm{E}$ & $12.11 \mathrm{~A}$ & $9.82 \mathrm{EFG}$ & $1.23 \mathrm{BC}$ \\
\hline $0.2 \%$ & $51.81 \mathrm{D}$ & $11.62 \mathrm{ABC}$ & $9.52 \mathrm{GH}$ & $1.21 \mathrm{~B}$ & $50.22 \mathrm{BC}$ & $11.20 \mathrm{AB}$ & $8.00 \mathrm{lJ}$ & $1.40 \mathrm{~B}$ \\
\hline $10 \mathrm{Kr}$ & $36.10 \mathrm{~L}$ & $7.72 \mathrm{H}$ & $14.72 \mathrm{~A}$ & $0.52 \mathrm{~J}$ & $34.28 \mathrm{~K}$ & $7.43 \mathrm{EF}$ & $15.45 \mathrm{~A}$ & $0.47 \mathrm{~J}$ \\
\hline $20 \mathrm{Kr}$ & $42.08 \mathrm{~J}$ & 9.84 DEF & $12.43 \mathrm{CD}$ & $0.78 \mathrm{GH}$ & $40.77 \mathrm{HI}$ & $9.23 \mathrm{BCDE}$ & $13.06 \mathrm{~B}$ & $0.70 \mathrm{HI}$ \\
\hline $30 \mathrm{Kr}$ & 43.57 I & $9.35 \mathrm{EFG}$ & $9.82 \mathrm{FG}$ & $0.94 \mathrm{DEF}$ & $42.75 \mathrm{FGH}$ & $10.09 \mathrm{BC}$ & $11.07 \mathrm{DE}$ & $0.91 \mathrm{EFG}$ \\
\hline $40 \mathrm{Kr}$ & $45.80 \mathrm{GH}$ & $9.28 \mathrm{EFG}$ & $8.08 \mathrm{~J}$ & $1.14 \mathrm{~B}$ & 43.62 FGH & $8.63 \mathrm{CDEF}$ & $9.24 \mathrm{FGHI}$ & $0.99 \mathrm{DEF}$ \\
\hline
\end{tabular}

Any means within the same column followed by the same letter are not statistically different at the $5 \%$ level (Duncan's multiple range test).

\section{2-generation}

\section{Number of days for first pistillate flower:}

It is cleared from Table (3) that the mean values of all doses were nearly from control value in two cultivars. Range of distribution in all treatment progenies in M2-generation were mostly wider than that of the control.Coefficient of variation values in both cultivars for all treatment progenies were higher than the respective control.These results are in agreement with that those of Hassan (1991) 
Table (2): Effect of gamma rays and EMS on some yield traits of two summer

\begin{tabular}{|c|c|c|c|c|c|c|c|c|}
\hline Treatments & $\begin{array}{c}\text { Early } \\
\text { yield / } \\
\text { plant } \\
\text { ( fruit } \\
\text { number) }\end{array}$ & $\begin{array}{c}\text { Early } \\
\text { yield/ } \\
\text { plot } \\
(\mathbf{k g})\end{array}$ & $\begin{array}{c}\text { Total } \\
\text { yield/ } \\
\text { plant } \\
\text { (fruit } \\
\text { number) }\end{array}$ & $\begin{array}{l}\text { Total } \\
\text { yield / } \\
\text { plot } \\
(\mathbf{k g})\end{array}$ & $\begin{array}{c}\text { Early } \\
\text { yield / } \\
\text { plant } \\
\text { ( fruit } \\
\text { number) }\end{array}$ & $\begin{array}{c}\text { Early } \\
\text { yield/ plot } \\
\text { (kg) }\end{array}$ & $\begin{array}{c}\text { Total } \\
\text { yield/ } \\
\text { plant } \\
\text { (fruit } \\
\text { number) }\end{array}$ & $\begin{array}{c}\text { Total } \\
\text { yield / } \\
\text { plot } \\
(\mathbf{k g})\end{array}$ \\
\hline Eskandarani & \multicolumn{4}{|c|}{2004} & \multicolumn{4}{|c|}{2005} \\
\hline \begin{tabular}{|l|} 
Control \\
\end{tabular} & $4.50 \mathrm{E}$ & $8.21 \mathrm{AB}$ & 11.27 DE & $20.63 \mathrm{AB}$ & $4.14 \mathrm{DE}$ & $7.74 \mathrm{~B}$ & $10.71 \mathrm{CD}$ & $20.00 \mathrm{~B}$ \\
\hline $0.025 \%$ & $4.31 \mathrm{EF}$ & $7.55 \mathrm{BC}$ & $10.30 \mathrm{EF}$ & $18.04 \mathrm{BCD}$ & $4.00 \mathrm{DEF}$ & $7.05 \mathrm{BC}$ & $9.41 \mathrm{DE}$ & $16.55 \mathrm{C}$ \\
\hline $0.05 \%$ & $3.90 \mathrm{GHI}$ & $6.65 \mathrm{CDE}$ & $9.01 \mathrm{GH}$ & 15.22 DEF & $3.85 \mathrm{EFG}$ & $6.74 \mathrm{C}$ & $7.57 \mathrm{EF}$ & $13.80 \mathrm{DE}$ \\
\hline $0.1 \%$ & $3.22 \mathrm{~K}$ & 5.07 FGH & $7.49 \mathrm{IJ}$ & $12.52 \mathrm{FG}$ & $3.25 \mathrm{HI}$ & $5.21 \mathrm{DEF}$ & $6.00 \mathrm{FGH}$ & $10.51 \mathrm{FG}$ \\
\hline $0.2 \%$ & $2.99 \mathrm{~K}$ & $4.05 \mathrm{I}$ & $6.00 \mathrm{~K}$ & $8.83 \mathrm{HIJ}$ & 3.021 & $4.65 \mathrm{FG}$ & $4.41 \mathrm{H}$ & $7.62 \mathrm{H}$ \\
\hline $10 \mathrm{Kr}$ & $5.21 \mathrm{BC}$ & $9.04 \mathrm{~A}$ & $13.01 \mathrm{~B}$ & $22.98 \mathrm{~A}$ & $4.98 \mathrm{~B}$ & $8.97 \mathrm{~A}$ & $12.50 \mathrm{BC}$ & $22.55 \mathrm{~A}$ \\
\hline $20 \mathrm{Kr}$ & $4.09 \mathrm{FGH}$ & $6.83 \mathrm{CD}$ & $10.30 \mathrm{EF}$ & $17.06 \mathrm{CD}$ & $4.15 \mathrm{DE}$ & $7.14 \mathrm{BC}$ & $9.33 \mathrm{DE}$ & $16.03 \mathrm{CD}$ \\
\hline $30 \mathrm{Kr}$ & $3.67 \mathrm{IJ}$ & $5.63 \mathrm{EFG}$ & $8.24 \mathrm{GHI}$ & $12.62 \mathrm{FG}$ & $3.25 \mathrm{HI}$ & $5.48 \mathrm{DE}$ & $7.44 \mathrm{EFG}$ & $13.41 \mathrm{E}$ \\
\hline $40 \mathrm{Kr}$ & $3.12 \mathrm{~K}$ & $4.57 \mathrm{HI}$ & $4.39 \mathrm{~L}$ & $8.29 \mathrm{IJ}$ & $2.96 \mid$ & $4.62 \mathrm{FG}$ & $5.46 \mathrm{GH}$ & $9.10 \mathrm{GH}$ \\
\hline \multicolumn{9}{|l|}{ Gabla } \\
\hline Control & $5.49 \mathrm{~B}$ & $7.47 \mathrm{BC}$ & $13.49 \mathrm{AB}$ & 15.97 CDE & $5.01 \mathrm{~B}$ & $7.16 \mathrm{BC}$ & $14.14 \mathrm{AB}$ & $20.18 \mathrm{~B}$ \\
\hline $0.025 \%$ & $5.08 \mathrm{C}$ & $6.69 \mathrm{CD}$ & $12.66 \mathrm{BC}$ & $16.73 \mathrm{CDE}$ & $4.86 \mathrm{~B}$ & $6.52 \mathrm{C}$ & $12.23 \mathrm{C}$ & $16.65 \mathrm{C}$ \\
\hline $0.05 \%$ & $4.58 \mathrm{DE}$ & $5.99 \mathrm{DEF}$ & $12.35 \mathrm{BCD}$ & 15.11 DEF & $4.51 \mathrm{C}$ & $5.73 \mathrm{D}$ & $11.04 \mathrm{CD}$ & $13.88 \mathrm{DE}$ \\
\hline $0.1 \%$ & $4.02 \mathrm{FGHI}$ & $5.58 \mathrm{EFG}$ & $9.23 \mathrm{FG}$ & $11.14 \mathrm{GH}$ & $3.98 \mathrm{EF}$ & $4.72 \mathrm{EFG}$ & $6.24 \mathrm{FGH}$ & $10.30 \mathrm{FG}$ \\
\hline $0.2 \%$ & $3.34 \mathrm{JK}$ & $4.38 \mathrm{HI}$ & $7.85 \mathrm{HIJ}$ & $6.74 \mathrm{~J}$ & $3.52 \mathrm{GH}$ & $4.09 \mathrm{G}$ & $5.95 \mathrm{FGH}$ & $7.57 \mathrm{H}$ \\
\hline $10 \mathrm{Kr}$ & $5.97 \mathrm{~A}$ & $7.32 \mathrm{BC}$ & $14.45 \mathrm{~A}$ & $18.59 \mathrm{BC}$ & $5.54 \mathrm{~A}$ & $7.80 \mathrm{~B}$ & $15.23 \mathrm{~A}$ & $21.40 \mathrm{AB}$ \\
\hline $20 \mathrm{Kr}$ & $4.90 \mathrm{CD}$ & $6.07 \mathrm{DEF}$ & $11.66 \mathrm{CD}$ & $13.93 \mathrm{EFG}$ & $4.37 \mathrm{CD}$ & $5.72 \mathrm{D}$ & $12.13 \mathrm{C}$ & $15.79 \mathrm{CD}$ \\
\hline $30 \mathrm{Kr}$ & $4.25 \mathrm{EFG}$ & $4.73 \mathrm{GHI}$ & $8.71 \mathrm{GHI}$ & $9.70 \mathrm{HI}$ & $3.96 \mathrm{EF}$ & $4.86 \mathrm{EFG}$ & $9.16 \mathrm{DE}$ & $12.10 \mathrm{EF}$ \\
\hline $40 \mathrm{Kr}$ & $3.37 \mathrm{HI}$ & \begin{tabular}{|l|}
$3.95 \mathrm{I}$ \\
\end{tabular} & $6.80 \mathrm{JK}$ & $7.15 \mathrm{IJ}$ & $3.71 \mathrm{FG}$ & $4.11 \mathrm{G}$ & $7.18 \mathrm{FG}$ & $8.95 \mathrm{GH}$ \\
\hline
\end{tabular}

Any means within the same column followed by the same letter are not statistically different at the $5 \%$ level (Duncan's multiple range test).

Table (3): Statistical parameter of number of days for first pistillate flower M2-generation for two summer squash cultivars.

\begin{tabular}{|c|c|c|c|c|c|c|c|c|c|c|c|c|}
\hline \multirow{2}{*}{ cultivar } & \multirow{2}{*}{ treatment } & \multirow{2}{*}{$\begin{array}{l}\text { No.of } \\
\text { plants }\end{array}$} & \multicolumn{7}{|c|}{ Classes } & \multirow{2}{*}{ Range } & \multirow{2}{*}{$X+$ S.E } & \multirow{2}{*}{ C.V\% } \\
\hline & & & $25-30$ & $31-35$ & $36-40$ & $41-45$ & $46-50$ & $51-55$ & $56-60$ & & & \\
\hline & Control 0.00 & 120 & & & & 87 & 33 & & & $42-47$ & $44.70+-011$ & 2.63 \\
\hline \multirow{11}{*}{ Eskandrani } & $\begin{array}{c}\text { Camma } \\
\text { iriadiation doses }\end{array}$ & & & & & & & & & & & \\
\hline & $10 \mathrm{Kr}$ & 100 & & 6 & & 58 & 33 & 3 & & $32-53$ & $44.28+-0.35$ & 7.90 \\
\hline & $20 \mathrm{Kr}$ & 120 & & 8 & 1 & 98 & 10 & 3 & & $31-54$ & $43.51+-0.32$ & 8.04 \\
\hline & $30 \mathrm{Kr}$ & 140 & 3 & 8 & 1 & 80 & 42 & 4 & 2 & $30-57$ & $44.21+-0.38$ & 10.04 \\
\hline & $40 \mathrm{Kr}$ & 120 & & & 4 & 54 & 42 & 15 & 5 & $39-57$ & $46.17+-0.36$ & 8.53 \\
\hline & $\begin{array}{c}\text { EMS } \\
\text { concentration } \\
\end{array}$ & & & & & & & & & & & \\
\hline & $0.025 \%$ & 140 & & & 13 & 76 & 51 & & & $38-48$ & $44.44+-0.19$ & 5.17 \\
\hline & $0.05 \%$ & 150 & 1 & 6 & & 119 & 22 & 2 & & $30-53$ & $43.39+-0.26$ & 7.39 \\
\hline & $0.1 \%$ & 120 & 3 & 9 & 13 & 57 & 38 & & & $30-49$ & $42.56+-0.40$ & 10.33 \\
\hline & $0.2 \%$ & 140 & & & 11 & 89 & 32 & 2 & 6 & $38-59$ & $44.28+-0.31$ & 8.42 \\
\hline & Control 0.00 & 140 & & & 19 & 121 & & & & $40-45$ & $42.00+-0.11$ & 2.67 \\
\hline \multirow{10}{*}{ Gabla } & $\begin{array}{c}\text { Camma } \\
\text { iriadiation doses }\end{array}$ & & & & & & & & & & & \\
\hline & $10 \mathrm{Kr}$ & 120 & 6 & 3 & & 111 & & & & $27-45$ & $41.30+-0.32$ & 8.49 \\
\hline & $20 \mathrm{Kr}$ & 100 & 3 & & 31 & 66 & & & & $28-44$ & $40.85+-0.24$ & 5.80 \\
\hline & $30 \mathrm{Kr}$ & 130 & 11 & & 33 & 86 & & & & $25-44$ & $40.14+-0.34$ & 9.69 \\
\hline & $40 \mathrm{Kr}$ & 140 & 2 & 2 & 30 & 101 & 4 & 1 & & $30-51$ & $41.47+-0.22$ & 6.39 \\
\hline & $\begin{array}{c}\text { EMS } \\
\text { concentration }\end{array}$ & & & & & & & & & & & \\
\hline & $0.025 \%$ & 120 & & & & 115 & 5 & & & $41-49$ & $42.56+-0.16$ & 4.08 \\
\hline & $0.05 \%$ & 140 & & & 40 & 95 & 5 & & & $37-48$ & $42.26+-0.22$ & 6.19 \\
\hline & $0.1 \%$ & 120 & 6 & 3 & 32 & 76 & 3 & & & $25-49$ & $40.64+-0.33$ & 8.95 \\
\hline & $0.2 \%$ & 150 & 4 & & 17 & 127 & 2 & & & $26-47$ & $42.53+-0.25$ & 7.12 \\
\hline
\end{tabular}

Number of nodes to first pistillate flower:

Data presented in Table (4) showed that there were little differences among all treatments and control in the two cultivars. The range of most treatments was wider than that of the control of two cultivars. The widest range was at $10 \mathrm{Kr}$ and $0.05 \%$ EMS (3-8) for Eskandarani cultivar and it was at $40 \mathrm{Kr}$ for Gabla cultivar (4-8). Coefficient of variation values in both 
cultivars for all treatment progenies were higher than the respective control. The maximum values of $\mathrm{CV} \%$ was obtained at $40 \mathrm{Kr}$ in Eskandarani cultivar $(14.40 \%)$ and at $0.2 \%$ EMS in Gabla cultivar (14.35).

Table (4):Statistical parameter of number of nodes for first pistillate flowers in M2- generation for two summer squash cultivars.

\begin{tabular}{|c|c|c|c|c|c|c|c|c|c|c|c|c|}
\hline \multirow{2}{*}{ cultivar } & \multirow{2}{*}{ treatment } & \multirow{2}{*}{$\begin{array}{l}\text { No.of } \\
\text { plants }\end{array}$} & \multicolumn{7}{|c|}{ Classes } & \multirow{2}{*}{ Range } & \multirow{2}{*}{$X \pm S . E$} & \multirow{2}{*}{ C.V\% } \\
\hline & & & 2 & 3 & 4 & 5 & 6 & 7 & 8 & & & \\
\hline & Control 0.00 & 120 & & & & 21 & 99 & & & $5-6$ & $5.83+-0.034$ & 6.51 \\
\hline \multirow{11}{*}{ Eskandrani } & $\begin{array}{c}\text { Camma iriadiation } \\
\text { doses }\end{array}$ & & & & & & & & & & & \\
\hline & $10 \mathrm{Kr}$ & 100 & & 5 & & 64 & 29 & 1 & 1 & $3-8$ & $5.24+-0.075$ & 14.31 \\
\hline & $20 \mathrm{Kr}$ & 120 & & & & 26 & 94 & & & $5-6$ & $5.78+-0.037$ & 7.09 \\
\hline & $30 \mathrm{Kr}$ & 140 & & & & 36 & 104 & & & $5-6$ & $5.74+-0.037$ & 7.66 \\
\hline & $40 \mathrm{Kr}$ & 120 & & 7 & & 42 & 70 & 1 & & $3-7$ & $5.48+-0.072$ & 14.41 \\
\hline & EMS concentration & & & & & & & & & & & \\
\hline & \begin{tabular}{|l|}
$0.025 \%$ \\
\end{tabular} & 140 & & & & 62 & 78 & & & $5-6$ & $5.56+-0.042$ & 8.99 \\
\hline & $0.05 \%$ & 150 & & 2 & 6 & 71 & 70 & & 1 & $3-8$ & $5.42+-0.54$ & 12.36 \\
\hline & $0.1 \%$ & 120 & & & 1 & 25 & 94 & & & $4-6$ & $5.78+-0.040$ & 7.61 \\
\hline & $0.2 \%$ & 140 & 3 & 4 & & 114 & 19 & & & $2-6$ & $5.01+-0.056$ & 13.37 \\
\hline & Control 0.00 & 140 & & & 60 & 80 & & & & $4-5$ & $4.57+-0.42$ & 10.94 \\
\hline \multirow{10}{*}{ Gabla } & $\begin{array}{c}\text { Camma iriadiation } \\
\text { doses }\end{array}$ & & & & & & & & & & & \\
\hline & $10 \mathrm{Kr}$ & 120 & 7 & & 4 & 115 & & 1 & & $4-7$ & $4.98+-0.23$ & 5.22 \\
\hline & $20 \mathrm{Kr}$ & 100 & & 4 & 31 & 58 & & & & $2-5$ & $4.40+-0.086$ & 17.04 \\
\hline & $30 \mathrm{Kr}$ & 130 & & 5 & 95 & 30 & & & & $3-5$ & $4.19+-0.042$ & 11.45 \\
\hline & $40 \mathrm{Kr}$ & 140 & & & 60 & 78 & & 1 & 1 & $4-8$ & $4.61+-0.051$ & 13.23 \\
\hline & EMS concentration & & & & & & & & & & & \\
\hline & \begin{tabular}{|l|}
$0.025 \%$ \\
\end{tabular} & 120 & 3 & & 26 & 93 & & 1 & & $4-7$ & $4.80+-0.042$ & 9.58 \\
\hline & $0.05 \%$ & 140 & & & 54 & 83 & & & & $2-5$ & $4.55+-0.052$ & 13.61 \\
\hline & $0.1 \%$ & 120 & 4 & & 84 & 36 & & & & $4-5$ & $4.30+-0.042$ & 10.69 \\
\hline & $0.2 \%$ & 150 & 4 & 2 & 97 & 47 & & & & $2-5$ & $4.25+-0.050$ & 14.35 \\
\hline
\end{tabular}

Number of pistillate flowers per plant:

As shown in Table (5), the number of pistillate flowers per plant recorded a little increase in all treatments compared with control of two cultivars. The range of most treatments was wider than that of the control. The coefficient of variation values in both cultivars for most treatments were higher than the respective control. Some treatments recorded lower $\mathrm{CV} \%$ values than the control, this case may be attributed to environmental conditions.

\section{Fruit weight:}

A significant difference between control and most treatments was observed in both cultivars (Table 6). All treatments produced lower weight of fruit compared to control. The maximum decrease was achieved by using 40 $\mathrm{Kr}$ of gamma-rays in both cultivars, it was 77.62 and $60.62 \mathrm{~g}$ for Eskandarani and Gabla cultivars, respectively. From the same Table it is clear that there were no differences between the effect of gamma-rays and EMS concentration. The SE (standard error) values were increased by increasing the doses of two mutagenesis. Concerning variation in fruit weight, all populations of $\mathrm{M} 2$ recorded relatively high $\mathrm{CV} \%$ values indicating high degree of heterogeneity in this respect. Moreover, the coefficient of variation of each mutagenized progenies was higher than the control. The maximum increase in variability of fruit weight was achieved at 30 and $40 \mathrm{Kr}$ gamma- 
rays for Eskandarani and Gabla cultivar, respectively. These results are similar to that found by Tickoo (1987).

Table (5): Statistical parameter of number of pistillate flowers / plant in M2- generation for two summer squash cultivars.

\begin{tabular}{|c|c|c|c|c|c|c|c|c|c|}
\hline \multirow{2}{*}{ cultivar } & \multirow{2}{*}{ treatment } & \multirow{2}{*}{$\begin{array}{l}\text { No.of } \\
\text { plants }\end{array}$} & \multicolumn{4}{|c|}{\begin{tabular}{|c|} 
Classes \\
\end{tabular}} & \multirow{2}{*}{ Range } & \multirow{2}{*}{ X+. S.E } & \multirow{2}{*}{ C.V\% } \\
\hline & & & $5-8$ & $9-12$ & 13-16 & $17-20$ & & & \\
\hline \multirow{12}{*}{ Eskandrani } & $\begin{array}{c}\text { Control } \\
0.00\end{array}$ & 60 & 7 & 42 & 11 & & $6-14$ & $10.66+-0.25$ & 18.75 \\
\hline & $\begin{array}{c}\text { Camma } \\
\text { iriadiation } \\
\text { doses }\end{array}$ & & & & & & & & \\
\hline & $10 \mathrm{Kr}$ & 50 & 3 & 37 & 9 & 2 & $8-19$ & $11.38+-0.31$ & 19.24 \\
\hline & $20 \mathrm{Kr}$ & 60 & 2 & 37 & 18 & 3 & $8-19$ & $12.03+-0.27$ & 17.70 \\
\hline & $30 \mathrm{Kr}$ & 70 & 5 & 40 & 24 & 1 & $8-18$ & $11.75+-0.27$ & 19.23 \\
\hline & \begin{tabular}{|l|}
$40 \mathrm{Kr}$ \\
\end{tabular} & 60 & 5 & 40 & 14 & 1 & $7-17$ & $11.48+-0.24$ & 16.55 \\
\hline & \begin{tabular}{|c|} 
EMS \\
concentration \\
\end{tabular} & & & & & & & & \\
\hline & \begin{tabular}{|l|}
$0.025 \%$ \\
\end{tabular} & 70 & 2 & 52 & 12 & 4 & $8-18$ & $11.58+-0.26$ & 18.91 \\
\hline & $0.05 \%$ & 75 & 6 & 35 & 33 & 1 & $5-17$ & $12.30+-0.28$ & 20.00 \\
\hline & $0.1 \%$ & 60 & 2 & 52 & 6 & & $6-14$ & $10.96+-0.18$ & 12.95 \\
\hline & $0.2 \%$ & 70 & & 50 & 20 & & $9-14$ & $11.82+-0.17$ & 12.43 \\
\hline & Control 0.00 & 70 & & 35 & 34 & 1 & $9-17$ & $12.98+-0.20$ & 13.09 \\
\hline \multirow{10}{*}{ Gabla } & $\begin{array}{c}\text { Camma } \\
\text { iriadiation } \\
\text { doses }\end{array}$ & & & & & & & & \\
\hline & $10 \mathrm{Kr}$ & 60 & 7 & 13 & 37 & 3 & $5-20$ & $12.95+-0.37$ & 22.70 \\
\hline & $20 \mathrm{Kr}$ & 50 & 1 & 14 & 33 & 2 & $8-21$ & $13.48+-0.29$ & 15.43 \\
\hline & $30 \mathrm{Kr}$ & 65 & & 20 & 42 & 3 & $10-20$ & $13.76+-0.23$ & 13.51 \\
\hline & \begin{tabular}{|c|}
$40 \mathrm{Kr}$ \\
\end{tabular} & 70 & 4 & 41 & 23 & 2 & $5-17$ & $11.90+-0.28$ & 20.00 \\
\hline & \begin{tabular}{|c|} 
EMS \\
concentration
\end{tabular} & & & & & & & & \\
\hline & \begin{tabular}{|l|}
$0.025 \%$ \\
\end{tabular} & 60 & 1 & 51 & 8 & & $8-15$ & $10.93+-018$ & 12.90 \\
\hline & $0.05 \%$ & 70 & 4 & 49 & 16 & 1 & $6-19$ & $11.71+-0.25$ & 18.44 \\
\hline & $0.1 \%$ & 60 & 8 & 35 & 16 & 1 & $5-19$ & $11.25+-0.36$ & 24.88 \\
\hline & $0.2 \%$ & 75 & 5 & 43 & 25 & 2 & $6-17$ & $12.05+-0.26$ & 19.00 \\
\hline
\end{tabular}

\section{Number of fruits per plant:}

It is clear from Table (7) that all treatments recorded higher mean values compared with control of two cultivars. The highest value was recorded at $0.05 \%$ EMS in Eskandrani cultivar (11.44 fruit) and it was at $30 \mathrm{Kr}$ in Gabla cultivar (12.49 fruit). Range of distribution of progenies of all treatments for both cultivars was slightly wider than that of the control. Coefficient of variation values in all progenies of most treatments within each cultivar were higher than the respective control value. The highest variation was found at $0.05 \%$ EMS and $30 \mathrm{Kr}$ in Eskandarani cultivar and it was at $0.1 \% \mathrm{EMS}$ and $10 \mathrm{Kr}$ in Gabla cultivar. Similar results were found by Hassan (1991), Ahmad and Yagoob (1993) and Srivastava and Singh (1996).

M3-generation:

The best mutations were selected depended on some commercial characters as early and high yield and data in Tables (8 and 9) show that each mutant was superior in one character comparative with control and other genotypes. EPF1 and GPF2 mutants are superior in number of pistillate flowers, EDN3 and GDN5 mutants were earlier than control and other genotypes, ENN4 and GNN5 mutants recorded the lowest number of nodes 
to first pistillate flower and EFW1 and GFW1 had the highest fruit weight. It can be concluded that these mutants have good and important characters and they are very important for breeding programs.

Table (6): Statistical parameter of average of fruit weight (g) in M2generation for two summer squash cultivars.

\begin{tabular}{|c|c|c|c|c|c|c|c|c|c|c|c|c|c|}
\hline \multirow{2}{*}{ cultivar } & \multirow{2}{*}{ treatment } & \multirow{2}{*}{\begin{tabular}{l|} 
No.of \\
plants
\end{tabular}} & \multicolumn{8}{|c|}{\begin{tabular}{|c|} 
Classes \\
\end{tabular}} & \multirow{2}{*}{ Range } & \multirow{2}{*}{$X \pm S . E$} & \multirow{2}{*}{ C.V\% } \\
\hline & & & $40-50$ & $51-60$ & $61-70$ & $71-80$ & 81-90 & $91-100$ & $101-110$ & $111-120$ & & & \\
\hline \multirow{11}{*}{ Eskandrani } & Control 0.00 & 60 & & & & 2 & 29 & 29 & & & 78-99 & $\begin{array}{c}90.76+- \\
0.69\end{array}$ & 5.66 \\
\hline & $\begin{array}{c}\text { Camma } \\
\text { iriadiation } \\
\text { doses }\end{array}$ & & & & & & & & & & & & \\
\hline & $10 \mathrm{Kr}$ & 50 & & & & 3 & 34 & 12 & & 1 & $80-120$ & $\begin{array}{c}88.42+- \\
1.00\end{array}$ & 8.00 \\
\hline & $20 \mathrm{Kr}$ & 60 & & & 2 & 14 & 32 & 11 & & 1 & $70-117$ & $\begin{array}{c}85.45+- \\
1.04\end{array}$ & 9.50 \\
\hline & $30 \mathrm{Kr}$ & 70 & & & 5 & 45 & 13 & 7 & & & $65-96$ & $\begin{array}{c}79.60+- \\
0.64 \\
\end{array}$ & 9.92 \\
\hline & $40 \mathrm{Kr}$ & 60 & & 10 & 11 & 16 & 14 & 9 & & & 55-99 & $\begin{array}{c}77.62+- \\
1.68\end{array}$ & 16.73 \\
\hline & \begin{tabular}{|c|} 
EMS \\
concentration
\end{tabular} & & & & & & & & & & & & \\
\hline & $0.025 \%$ & 70 & & & & 6 & 36 & 28 & & & $71-100$ & $\begin{array}{c}89.69+- \\
0.76\end{array}$ & 7.16 \\
\hline & $0.05 \%$ & 75 & & & 3 & 15 & 27 & 8 & & & $67-98$ & $\begin{array}{c}84.46+- \\
0.72 \\
\end{array}$ & 7.43 \\
\hline & $0.1 \%$ & 60 & & 5 & 7 & 16 & 25 & 7 & & & 58-95 & $\begin{array}{c}80.96+- \\
1.36\end{array}$ & 13.04 \\
\hline & $0.2 \%$ & 70 & & 7 & 14 & 17 & 23 & 9 & & & $55-100$ & $\begin{array}{c}78.18+- \\
1.39 \\
\end{array}$ & 14.91 \\
\hline & Control 0.00 & 70 & & & 44 & 26 & & & & & $62-76$ & $\begin{array}{c}68.98+- \\
0.55\end{array}$ & 6.75 \\
\hline \multirow{10}{*}{ Gabla } & $\begin{array}{c}\text { Camma } \\
\text { iriadiation } \\
\text { doses }\end{array}$ & & & & & & & & & & & & \\
\hline & $10 \mathrm{Kr}$ & 60 & & 7 & 42 & 10 & & 1 & & & 60-95 & $\begin{array}{c}67.17+- \\
0.68\end{array}$ & 7.87 \\
\hline & $20 \mathrm{Kr}$ & 50 & 1 & 13 & 36 & & & & & & $50-70$ & $\begin{array}{c}63.72+- \\
0.73 \\
\end{array}$ & 8.12 \\
\hline & $30 \mathrm{Kr}$ & 65 & 7 & 13 & 41 & 4 & & & & & $48-73$ & $\begin{array}{c}62.81+- \\
0.87\end{array}$ & 11.22 \\
\hline & $40 \mathrm{Kr}$ & 70 & 17 & 10 & 42 & 1 & & & & & $45-71$ & $\begin{array}{c}60.62+- \\
1.08\end{array}$ & 14.89 \\
\hline & $\begin{array}{c}\text { EMS } \\
\text { concentration }\end{array}$ & & & & & & & & & & & & \\
\hline & $0.025 \%$ & 60 & & & 51 & 9 & & & & & $62-73$ & $\begin{array}{c}67.32+- \\
0.37\end{array}$ & 4.27 \\
\hline & $0.05 \%$ & 70 & & 6 & 60 & 4 & & & & & $53-73$ & $\begin{array}{c}66.02+- \\
0.51 \\
\end{array}$ & 6.52 \\
\hline & $0.1 \%$ & 60 & 7 & 12 & 37 & 4 & & & & & $48-72$ & $\begin{array}{c}62.84+- \\
0.93\end{array}$ & 11.55 \\
\hline & $0.2 \%$ & 75 & 12 & 7 & 51 & 5 & & & & & $42-72$ & $\begin{array}{c}62.21+- \\
0.93\end{array}$ & 13.05 \\
\hline
\end{tabular}


El-Miniawy, S.M. et al.

Table (7): Statistical parameter of number of fruits / plant in M2generation for two summer squash cultivars.

\begin{tabular}{|c|c|c|c|c|c|c|c|c|c|c|}
\hline \multirow{2}{*}{ cultivar } & \multirow{2}{*}{ treatment } & \multirow{2}{*}{$\begin{array}{l}\text { No.of } \\
\text { plants }\end{array}$} & \multicolumn{5}{|c|}{ Classes } & \multirow{2}{*}{ Range } & \multirow{2}{*}{$X \pm S . E$} & \multirow{2}{*}{ C.V\% } \\
\hline & & & $3-6$ & $7-10$ & 11.13 & $14-17$ & $18-21$ & & & \\
\hline & Control 0.00 & 60 & 4 & 33 & 23 & & & $6-13$ & $9.90+-0.21$ & 16.76 \\
\hline \multirow{11}{*}{ Eskandrani } & $\begin{array}{c}\text { Camma iriadiation } \\
\text { doses }\end{array}$ & & & & & & & & & \\
\hline & $10 \mathrm{Kr}$ & 50 & & 28 & 19 & 3 & & $7-16$ & $\begin{array}{c}10.44+- \\
0.26\end{array}$ & 17.81 \\
\hline & $20 \mathrm{Kr}$ & 60 & & 27 & 29 & 4 & & $7-17$ & $\begin{array}{c}10.86+- \\
0.22\end{array}$ & 15.93 \\
\hline & $30 \mathrm{Kr}$ & 70 & 2 & 30 & 30 & 8 & & $6-17$ & $\begin{array}{c}10.77+- \\
0.26\end{array}$ & 20.42 \\
\hline & $40 \mathrm{Kr}$ & 60 & 3 & 25 & 30 & 2 & & $6-16$ & $\begin{array}{c}10.66+- \\
0.26\end{array}$ & 18.94 \\
\hline & EMS concentration & & & & & & & & & \\
\hline & $0.025 \%$ & 70 & & 41 & 22 & 7 & & $7-17$ & $\begin{array}{c}10.67+- \\
0.26\end{array}$ & 20.52 \\
\hline & $0.05 \%$ & 75 & 1 & 26 & 31 & 17 & & $3-16$ & $\begin{array}{c}11.44+- \\
0.28\end{array}$ & 21.67 \\
\hline & $0.1 \%$ & 60 & 2 & 37 & 21 & & & $4-13$ & $\begin{array}{c}10.08+- \\
0.18\end{array}$ & 14.48 \\
\hline & $0.2 \%$ & 70 & & 35 & 33 & 2 & & $7-14$ & $\begin{array}{c}10.68+- \\
0.17 \\
\end{array}$ & 13.57 \\
\hline & Control 0.00 & 70 & & 7 & 49 & 14 & & $9-16$ & $\begin{array}{l}12.14+- \\
0.18\end{array}$ & 12.16 \\
\hline \multirow{10}{*}{ Gabla } & $\begin{array}{c}\text { Camma iriadiation } \\
\text { doses }\end{array}$ & & & & & & & & & \\
\hline & $10 \mathrm{Kr}$ & 60 & 3 & 13 & 30 & 13 & 1 & $3-18$ & $\begin{array}{c}11.61+- \\
0.36\end{array}$ & 24.03 \\
\hline & $20 \mathrm{Kr}$ & 50 & & 7 & 28 & 14 & 1 & $8-19$ & $\begin{array}{c}12.38+- \\
0.28\end{array}$ & 16.23 \\
\hline & $30 \mathrm{Kr}$ & 65 & & 11 & 32 & 21 & 1 & $8-18$ & $\begin{array}{c}12.49+- \\
0.23 \\
\end{array}$ & 15.37 \\
\hline & $40 \mathrm{Kr}$ & 70 & 2 & 32 & 27 & 9 & & $4-16$ & $\begin{array}{c}10.47+- \\
0.26\end{array}$ & 20.76 \\
\hline & EMS concentration & & & & & & & & & \\
\hline & $0.025 \%$ & 60 & & 34 & 17 & & & $7-13$ & $9.51+-0.18$ & 14.93 \\
\hline & $0.05 \%$ & 70 & 2 & 38 & 22 & 8 & & $4-15$ & $\begin{array}{c}10.47+^{+-} \\
0.26\end{array}$ & 20.72 \\
\hline & $0.1 \%$ & 60 & 8 & 19 & 26 & 7 & & $4-15$ & $\begin{array}{c}10.28+- \\
0.35\end{array}$ & 26.55 \\
\hline & $0.2 \%$ & 75 & 3 & 32 & 27 & 13 & & $5-16$ & $\begin{array}{l}10.97+- \\
0.27\end{array}$ & 21.69 \\
\hline
\end{tabular}


Table (8):Stem length, No. of leaves per plant, No. of staminate flowers/ plant, No. of pistillate flowers/ plant, No. of nodes to first pistillate flower and No. of days to first pistillate flower for 8 mutant lines of summer squash for Eskandarani and Gabla cultivars and their original cultivars in M3-generation

\begin{tabular}{|c|c|c|c|c|c|c|}
\hline genotype & $\begin{array}{c}\text { Stem } \\
\text { length } \\
\text { (cm) }\end{array}$ & $\begin{array}{c}\text { No. of } \\
\text { leaves/ } \\
\text { plant }\end{array}$ & $\begin{array}{c}\text { No. of } \\
\text { staminate } \\
\text { flowers/ } \\
\text { plant }\end{array}$ & $\begin{array}{c}\text { No. of } \\
\text { pistillate } \\
\text { flowers/ } \\
\text { plant }\end{array}$ & $\begin{array}{c}\text { No. of } \\
\text { nodes to } \\
\text { first } \\
\text { pistillate } \\
\text { flower }\end{array}$ & $\begin{array}{c}\text { No. of days } \\
\text { to first } \\
\text { pistillate } \\
\text { flower }\end{array}$ \\
\hline \multicolumn{7}{|c|}{ Eskandarani } \\
\hline Control & 84.36 & 21.65 & 14.07 & 11.35 & 5.22 & 45.67 \\
\hline EPF1 & 84.00 & 21.00 & 7.33 & 18.74 & 4.00 & 39.68 \\
\hline EDN3 & 81.33 & 18.33 & 9.65 & 13.00 & 4.66 & 30.03 \\
\hline ENN4 & 83.33 & 19.67 & 12.64 & 15.67 & 2.40 & 35.00 \\
\hline EFW1 & 92.33 & 23.00 & 12.60 & 11.60 & 5.03 & 44.31 \\
\hline L.S.D 5\% & 2.87 & 1.85 & 1.70 & 1.59 & 0.60 & 1.88 \\
\hline \multicolumn{7}{|c|}{ Gabla } \\
\hline Control & 71.35 & 17.11 & 14.02 & 11.32 & 4.15 & 41.65 \\
\hline GPF2 & 74.00 & 13.69 & 17.60 & 6.12 & 4.00 & 39.00 \\
\hline GDN5 & 68.00 & 20.22 & 17.00 & 5.28 & 3.66 & 27.03 \\
\hline GNN5 & 70.00 & 17.23 & 14.63 & 7.00 & 2.19 & 42.00 \\
\hline GFW1 & 76058 & 16.33 & 14.33 & 9.66 & 4.33 & 40.00 \\
\hline LSD 5\% & 2.692 & 1.425 & 1.402 & 1.544 & 0.540 & 1.799 \\
\hline
\end{tabular}

PF = high number of pistillate flowers mutant $\quad F W=$ Large and small fruit mutant

Table (9):Fruit length, diameter, weight, and number per plant and 100 seed weight for 8 mutant lines of summer squash cv. Eskandarani and Gabla cultivars and their original cultivars in M3-generation.

\begin{tabular}{|cccccc|}
\hline genotype & $\begin{array}{c}\text { Fruit length } \\
(\mathrm{cm})\end{array}$ & $\begin{array}{c}\text { Fruit diameter } \\
(\mathrm{cm})\end{array}$ & $\begin{array}{c}\text { Average fruit } \\
\text { weight } \\
(\mathrm{g})\end{array}$ & $\begin{array}{c}\text { Fruit number / } \\
\text { plant }\end{array}$ & $\begin{array}{c}100 \text { seeds } \\
\text { weight } \\
(\mathrm{g})\end{array}$ \\
\hline Control & 13.33 & 2.65 & 88.35 & 10.33 & 17.87 \\
\hline EPF1 & 13.30 & 2.36 & 84.00 & 17.00 & 17.00 \\
\hline EDN3 & 14.12 & 2.73 & 85.00 & 12.67 & 12.12 \\
\hline ENN4 & 11.00 & 2.80 & 87.00 & 14.67 & 17.67 \\
\hline EFW1 & 15.33 & 3.33 & 117.60 & 11.00 & 17.26 \\
\hline L.S.D 5\% & 1.26 & 0.19 & 1.55 & 2.11 & 1.59 \\
\hline & & & Gabla & & 13.37 \\
\hline Control & 7.66 & 3.20 & 70.45 & 16.67 & 16.67 \\
\hline GPF2 & 9.14 & 3.40 & 71.60 & 15.33 & 14.00 \\
\hline GDN5 & 7.57 & 3.13 & 67.00 & 13.00 & 13.67 \\
\hline GNN5 & 6.33 & 3.46 & 58.67 & 13.67 & 16.00 \\
\hline GFW1 & 9.66 & 3.33 & 94.56 & 1.476 & 1.366 \\
\hline LSD 5\% & 1.038 & 0.197 & 1.813 & & \\
\hline
\end{tabular}

DN = Early flowering mutant

$\mathrm{NN}=$ node position mutant

$\mathrm{PF}=$ high number of pistillate flowers mutant $\quad \mathrm{FW}=$ Large and small fruit mutant 


\section{REFERENCES}

Abd El-Maksoud, B.A. (1980). Effect of gamma irradiation on Portulaca grandiflora Hook. M. Sci. Fac. Agric., Alex. Univ.

Ahmad, B. and M. Yagoob. (1993). Radiation for induced mutation in mungbean (Vigna radiate L. Wilezek). Sarhad Journal of Agriculture. (1993). 9 (5) : 423-427. (C. F. PI. Breed. Abst. 65, 529, 1995.).

Hassan, H. R. (1991). Induced variability in morphological and reproductive characteristics of Lupinus termis. Frosk. By chemical mutagenesis. M.Sc. Thesis, Fac. Agric. Cairo Univ.

Kumar, Y. and V. K. Mishra. (1999). Effect of gamma-rays and diethyl sulphate on germination, growth fertility and yield in green gram Vigna radiate L. Eilezek. Annals of Agric. Res. 20 (2) : 144-147.

Nofal, F. A. (1989). Effect of gamma irradiation on morphological and reproductive characters of two pea cultivars. J. Agric. Sci. Mansoura. Univ. 14 (2): 599-609, 1989.

Saxena, P. K and Solanki, S.S. (1986) Effect of gamma radiation on growth and yield of Lycopersicon esculentum Mill. Prog Hortic 18 : (3-4). 178180. (C. F. PI Breed. Abst. 55 (12) : 11158, 1986).

Shalaby, G. I. and A. M. Nassar and M A. Farghaly (1983). Effect of EMS on yield and Quality of Egyptian garlic. Agric. Sci. Assuit. 14 (4) : 15-26.

Srivastava, A. and V. P. Singh. (1996). Induced high yielding pigeon pea mutants. Mutation Breeding Newsletter. Vienna. Issue. 42 (6). : 7-9.

Tickoo, J. L. (1987). Spectrum and frequency of induced macromutations in mung bean. Crop Improvement Society of India.13.(2): 116-117. (C. F. PI Breed. Abst. 57 (12) : 11278, 1987).

Waller, R.A. and D.B Duncan (1960). Ways for the symmetric multiple comparison. Problem Amer. Stat. Assoc. J. 19: 1485-1503.

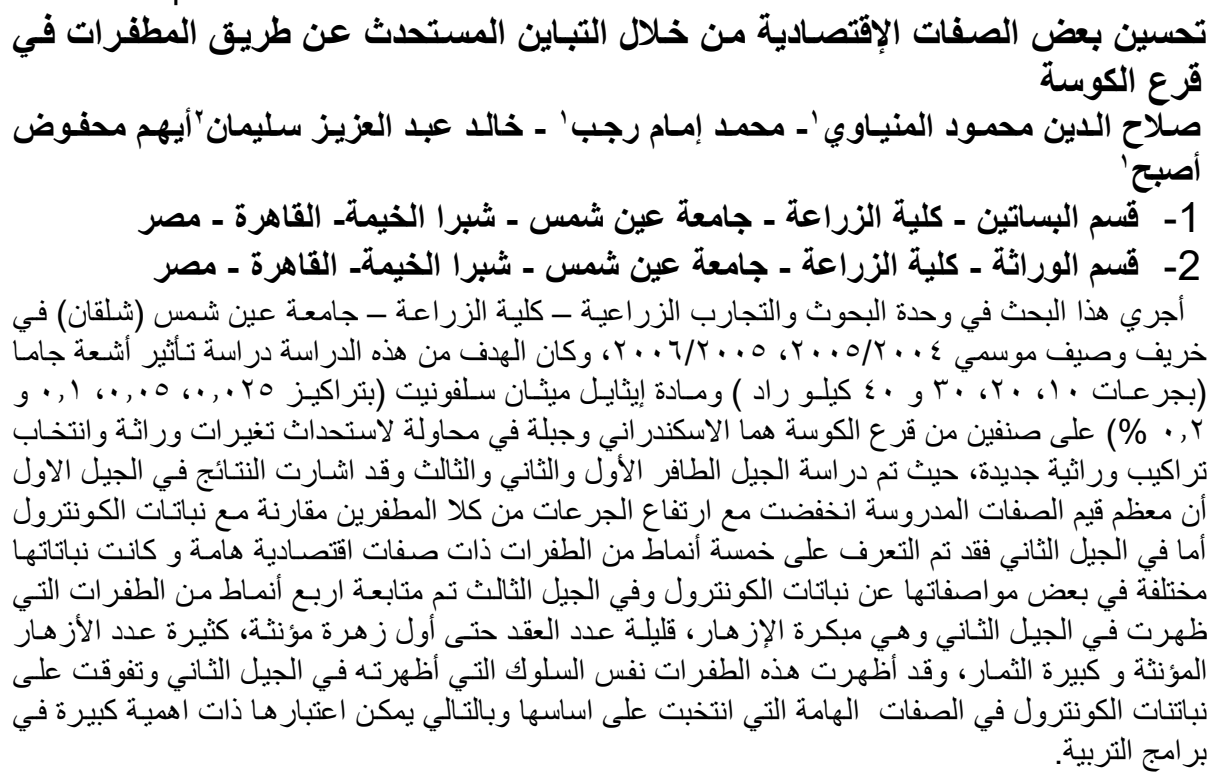

